\title{
Video Article \\ Preparation of Chloroplast Sub-compartments from Arabidopsis for the Analysis of Protein Localization by Immunoblotting or Proteomics
}

\author{
Imen Bouchnak ${ }^{1}$, Lucas Moyet ${ }^{1}$, Daniel Salvi ${ }^{1}$, Marcel Kuntz ${ }^{1}$, Norbert Rolland ${ }^{1}$ \\ ${ }^{1}$ Université Grenoble Alpes, INRA, CNRS, CEA
}

Correspondence to: Norbert Rolland at norbert.rolland@cea.fr

URL: https://www.jove.com/video/58581

DOI: doi: $10.3791 / 58581$

Keywords: Biochemistry, Issue 140, Plant, Arabidopsis, chloroplast, organelle, plastid, envelope, stroma, thylakoid, cell fractionation, Percoll, localization, immunoblotting

Date Published: 10/19/2018

Citation: Bouchnak, I., Moyet, L., Salvi, D., Kuntz, M., Rolland, N. Preparation of Chloroplast Sub-compartments from Arabidopsis for the Analysis of Protein Localization by Immunoblotting or Proteomics. J. Vis. Exp. (140), e58581, doi:10.3791/58581 (2018).

\section{Abstract}

Chloroplasts are major components of plant cells. Such plastids fulfill many crucial functions, such as assimilation of carbon, sulfur and nitrogen as well as synthesis of essential metabolites. These organelles consist of the following three key sub-compartments. The envelope, characterized by two membranes, surrounds the organelle and controls the communication of the plastid with other cell compartments. The stroma is the soluble phase of the chloroplast and the main site where carbon dioxide is converted into carbohydrates. The thylakoid membrane is the internal membrane network consisting of grana (flat compressed sacs) and lamellae (less dense structures), where oxygenic photosynthesis takes place. The present protocol describes step by step procedures required for the purification, using differential centrifugations and Percoll gradients, of intact chloroplasts from Arabidopsis, and their fractionation, using sucrose gradients, in three sub-compartments (i.e., envelope, stroma, and thylakoids). This protocol also provides instructions on how to assess the purity of these fractions using markers associated to the various chloroplast sub-compartments. The method described here is valuable for subplastidial localization of proteins using immunoblotting, but also for subcellular and subplastidial proteomics and other studies.

\section{Video Link}

The video component of this article can be found at https://www.jove.com/video/58581/

\section{Introduction}

Chloroplasts are major components of plant cells. They derive from a cyanobacterial ancestor that has undergone an endosymbiosis and eventually evolved as an organelle during evolution ${ }^{1,2}$. Such organelles contain three main compartments (Figure 1). The envelope system is made of an inner and an outer membranes surrounding the organelle. This double membrane system contains various enzymes involved in the metabolism of lipids and pigments and is mostly devoted to the control of the communication between plastids and the cytosol. It contains various transport systems that allow the import of nuclear-encoded proteins, and the exchange of ions and metabolites between the cytosol and the chloroplast thus regulating essential metabolic functions of the plant cell ${ }^{3,4}$. The stroma, the soluble phase of the chloroplast, contains enzymes of the Calvin cycle $\left(\mathrm{CO}_{2}\right.$ assimilation), the synthesis of various metabolites including amino acids and vitamins, and the transcription and translation machineries of the plastid. The thylakoid membrane is a widely organized internal membrane network where the light phase of photosynthesis takes place. Thereby, chloroplasts are the place where essential metabolic pathways occur ${ }^{5}$.

In order to decipher new regulatory mechanisms that control the chloroplast dynamics and physiology, defining the sub-plastidial localization of chloroplast proteins is thus critical to support targeted studies aiming to better understand proteins functions in model organisms ${ }^{6}$. In order to get access to the genuine subplastidial localization of these proteins, it is thus essential to start from highly pure subplastidial fractions (envelope membranes, stroma, and thylakoids). In this context, the aim of the present protocol is to purify intact chloroplasts from Arabidopsis leaves using differential centrifugations and continuous Percoll gradients, and to fractionate them using discontinuous sucrose gradients, in three subcompartments (i.e., envelope, stroma, and thylakoids). The method described here also provides instructions to assess the purity of purified suborganellar fractions using markers associated to the various chloroplast sub-compartments. This protocol is valuable for subplastidial localization of proteins using immunoblotting and for further analysis of purified fractions using mass spectrometry (MS)-based proteomic studies.

\section{Preparation of Buffers, Stock Solutions, and Gradients}

1. Prepare the following stock solutions that can be stored up to 6 months at $4{ }^{\circ} \mathrm{C}$.

1. Prepare $1 \mathrm{~L}$ of Tricine buffer $(1 \mathrm{M}, \mathrm{pH} 8.4)$ and Tricine buffer $(1 \mathrm{M}, \mathrm{pH} 7.6)$. Adjust $\mathrm{pH}$ by adding $\mathrm{KOH}$ pellets.

2. Prepare $1 \mathrm{~L}$ of ethylenediaminetetraacetic acid (EDTA, $0.5 \mathrm{M}, \mathrm{pH}$ 8) and 3-(N-morpholino) propane sulfonic acid (MOPS) buffer (1 M, $\mathrm{pH}$ 7.8). Adjust $\mathrm{pH}$ by adding $\mathrm{NaOH}$ pellets. 
3. Prepare $50 \mathrm{~mL}$ of $\mathrm{MgCl}_{2}(1 \mathrm{M})$.

4. Prepare $50 \mathrm{~mL}$ of protease inhibitors solutions: phenylmethylsulfonyl fluoride (PMSF set up in isopropanol, $100 \mathrm{mM}$ ), benzamidine hydrochloride hydrate $(100 \mathrm{mM})$, and $\varepsilon$-amino caproic acid $(50 \mathrm{mM})$.

NOTE: While PMSF and amino caproic acid are stable in solution for months at $4{ }^{\circ} \mathrm{C}$, benzamidine solution should be stored at $-20^{\circ} \mathrm{C}$.

2. Prepare the following solutions the day before the experiment and store all solutions at $4{ }^{\circ} \mathrm{C}$.

1. Prepare $4 \mathrm{~L}$ of grinding medium $\mathrm{pH} 8.4$ containing Tricine- $\mathrm{KOH}(20 \mathrm{mM}, \mathrm{pH} 8.4)$, sorbitol $(0.4 \mathrm{M})$, EDTA (10 mM, pH 8), and NaHCO 3 $(10 \mathrm{mM})$. Adjust $\mathrm{pH}$ by adding $\mathrm{NaOH}$ pellets. Add bovine serum albumin (BSA) at $0.1 \%(\mathrm{w} / \mathrm{v})$ just before use and mix well.

2. Prepare $500 \mathrm{~mL}$ of washing medium $(2 \mathrm{x}) \mathrm{pH} 7.6$ containing Tricine- $\mathrm{KOH}(20 \mathrm{mM}, \mathrm{pH} 7.6)$, sorbitol $(0.8 \mathrm{M}), \mathrm{MgCl}_{2}(5 \mathrm{mM})$, and EDTA $(2.5 \mathrm{mM})$. Adjust $\mathrm{pH}$ by adding $\mathrm{NaOH}$ pellets. Dilute such solution after preparation of Percoll gradient solution to obtain washing medium $(1 \mathrm{x})$.

3. Prepare $200 \mathrm{~mL}$ of Percoll gradient solution for chloroplast purification by mixing Percoll with washing medium $(2 \mathrm{x})$ at an equal volume to get a final solution at $50 \%(\mathrm{v} / \mathrm{v})$ Percoll / $0.4 \mathrm{M}$ sorbitol.

4. Prepare $50 \mathrm{~mL}$ of sucrose solutions for chloroplast fractionation by mixing MOPS (10 mM, $\mathrm{pH} 7.8), \mathrm{MgCl}_{2}(4 \mathrm{mM})$, and different concentrations of sucrose $(0.3 \mathrm{M}, 0.6 \mathrm{M}$, and $0.93 \mathrm{M})$.

3. Prepare the following gradients and buffers prior to starting the experiment.

1. Prepare six tubes of Percoll gradients (each containing $30 \mathrm{~mL}$ of a $50 \%$ Percoll / $0.4 \mathrm{M}$ sorbitol) by centrifugation at $38,700 \times \mathrm{g}$ for 55 min at $4{ }^{\circ} \mathrm{C}$. Keep the brake off to prevent blending of the gradients. After centrifugation, store the tubes containing the preformed gradients in a cold room until use.

2. Prepare four tubes of sucrose gradients, with each gradient formed of three following sucrose layers: $3 \mathrm{~mL}$ of $0.93 \mathrm{M}, 2.5 \mathrm{~mL}$ of $0.6 \mathrm{M}$, and $2 \mathrm{~mL}$ of $0.3 \mathrm{M}$ sucrose. Carefully overlay each layer, using a peristaltic pump starting with $0.93 \mathrm{M}$ at the bottom and finishing with $0.3 \mathrm{M}$ at the top.

3. Prepare $50 \mathrm{~mL}$ of hypotonic medium for chloroplast lysis containing MOPS (10 mM, pH 7.8), $\mathrm{MgCl}_{2}(4 \mathrm{mM}), \mathrm{PMSF}$ (1 mM, set up in isopropanol), benzamidine hydrochloride hydrate $(1 \mathrm{mM})$, and $\varepsilon$-amino caproic acid $(0.5 \mathrm{mM})$. Store the buffer on ice until use.

4. Prepare $50 \mathrm{~mL}$ of membrane washing buffer containing MOPS (10 mM, pH 7.8), PMSF (1 mM), benzamidine hydrochloride hydrate (1 $\mathrm{mM})$, and $\varepsilon$-amino caproic acid $(0.5 \mathrm{mM})$. Store the buffer on ice until use.

\section{Growth and Harvesting of Arabidopsis Leaves}

1. For growth of Arabidopsis plants, prepare 4 large plastic pans (for a total surface of 0.5 to $1 \mathrm{~m}^{2}$ ) of Arabidopsis plants by sowing $30 \mathrm{mg}$ of seeds in each pan. Grow Arabidopsis plants for 5 weeks at 12 -h light cycle at $23^{\circ} \mathrm{C}$ (day) $/ 18^{\circ} \mathrm{C}$ (night) with a light intensity of $150 \mu \mathrm{M} \mathrm{m}^{-2} \mathrm{~s}^{-1}$.

2. Incubate plants in a dark and cold room $\left(4^{\circ} \mathrm{C}\right)$ overnight prior to the experiment (to reduce the amount of starch granules in chloroplasts).

3. Pre-weigh a $1 \mathrm{~L}$ beaker and then place it on ice before starting harvesting of leaf material.

4. Harvest Arabidopsis leaves by avoiding soil (compost). Re-weigh the beaker and record the tissue weight. NOTE: 400 to $500 \mathrm{~g}$ of leaf material are expected from four pans.

5. Homogenize leaves in a cold room with $2 \mathrm{~L}$ of grinding buffer (add BSA before use) three times/2 s each time, in a blender at high speed.

6. Filter the homogenate in a cold room using 4 layers of muslin and one layer of nylon blutex. Gently squeeze the homogenate leaves inside the muslin/nylon blutex to extract all the liquid.

7. Recover the remaining tissue in the blender cup for a second extraction. Repeat steps 2.5 and 2.6 using $2 \mathrm{~L}$ of grinding medium and new $4-5$ layers of muslin (in a cold room).

\section{Purification of Crude Chloroplasts Using Differential Centrifugation}

1. Equally distribute the crude cell extract into six $500 \mathrm{~mL}$ bottles and place the bottles on ice before centrifugation. Centrifuge for 2 min as soon as the maximum speed $(2,070 \mathrm{xg})$ is reached (maximum acceleration and brake on, $4^{\circ} \mathrm{C}$ ).

2. Gently discard the supernatant.

3. Aspirate the remaining supernatant using a water pump and keep the pellets containing concentrated crude chloroplasts on ice.

4. Gently resuspend pellets by adding a minimal volume of washing medium $(1 \mathrm{x})$ (final volume of the combined chloroplast suspensions $=$ $36 \mathrm{~mL}$ ) using a paintbrush or a curved plastic spatula. Use a $10 \mathrm{~mL}$ pipet to add $3 \mathrm{~mL}$ of washing medium in each bottle. NOTE: Do not use pipet with very fine tips to avoid breakage of chloroplasts. Alternatively, cut the blue tip of a pipet with a razor blade to generate a larger hole.

5. Collect the resuspended chloroplasts in one tube by using a $10 \mathrm{~mL}$ pipet. Gently mix by inverting the tube to obtain a homogenous suspension prior to loading on Percoll gradients.

\section{Purification of Intact Chloroplasts on Continuous Percoll Gradient}

1. Slowly load $6 \mathrm{~mL}$ of the chloroplast suspension on top of each of the six Percoll gradients using a $10 \mathrm{~mL}$ pipet to avoid breakage of chloroplasts.

2. Centrifuge the gradients for $10 \mathrm{~min}$ at $13,300 \mathrm{xg}, 4^{\circ} \mathrm{C}$ using a swinging-bucket rotor.

NOTE: The acceleration should be slow, and the brake should be disconnected (brake off or slow deceleration) to prevent blending of the Percoll gradients.

3. Aspirate the upper phase that contains broken chloroplasts and intact mitochondria using a water pump, and then retrieve intact chloroplasts present in the lower phase (the broad dark-green band) with a $10 \mathrm{~mL}$ pipet. Be careful not to aspirate nuclei and cell debris (found at the bottom of the tube) with the intact chloroplasts (Figure 2A). 
4. Dilute 3-4-fold the intact chloroplast suspension with washing buffer $(1 \mathrm{x})$. Centrifuge for $2 \mathrm{~min}$ as soon as the maximum speed $(2,070 \mathrm{x} \mathrm{g}$, $4{ }^{\circ} \mathrm{C}$ ) is reached (maximum acceleration and brake on).

5. Carefully discard the supernatant.

6. Completely aspirate the remaining supernatant with a water pump and keep the pellet of concentrated intact chloroplasts on ice.

7. Before chloroplast lysis, keep an aliquot of intact chloroplast fraction in approximately $1 \mathrm{~mL}$ of washing medium $(1 \mathrm{x})$ for further analyses using sodium dodecyl sulfate polyacrylamide gel electrophoresis (SDS-PAGE) and western blotting. Keep a small aliquot of these intact chloroplasts for determination of protein concentration. Store the intact chloroplast fraction in liquid nitrogen for further experiments.

\section{Lysis of Intact Chloroplasts Using a Hypotonic Buffer and Purification of Chloroplast Sub-Compartments on Discontinuous Sucrose Gradients}

1. Lyse the purified intact chloroplasts by resuspending the pellet in hypotonic medium that contains protease inhibitors (the final volume should not exceed $12 \mathrm{~mL}$ ).

NOTE: From this step, the use of pipet with fine tips (blue tips) is possible since intactness of chloroplasts is no more essential (pipetting chloroplasts up and down as long as pellet is not entirely resuspended). Arabidopsis chloroplasts are very fragile (when compared to pea chloroplasts, for example) and their lysis is almost immediate after incubation in hypotonic medium.

2. Slowly load $3 \mathrm{~mL}$ of the lysed chloroplasts on the top of each preformed sucrose gradients using a peristaltic pump.

3. Ultracentrifuge the gradients for $1 \mathrm{~h}$ at $\left(70,000 \times \mathrm{g}, 4^{\circ} \mathrm{C}\right)$. Balance pairs of tubes using hypotonic medium buffer prior to centrifugation.

4. Carefully recover the soluble stromal proteins by pipetting the upper phase of the gradient ( $3 \mathrm{~mL}$ from each gradient) (Figure 2B). Take an aliquot for determination of protein concentration. Store the stroma in liquid nitrogen for further experiments.

5. Aspirate the remaining upper phase of each gradient up to the yellow band using a water pump.

6. Retrieve the yellow band (the envelope) with a pipet (approximately $1 \mathrm{~mL}$ from each gradient). Pool the envelopes in one tube.

7. Remove the remaining phase of each gradient up to the thylakoid pellet using a water pump.

\section{Washing and Concentration of Thylakoid and Envelope Membrane Systems}

1. Resuspend the thylakoid pellets (green pellets) in a minimum volume $(2 \mathrm{~mL})$ of membrane washing buffer $(1 \mathrm{x})$ (with protease inhibitors).

2. Dilute the envelope and thylakoid suspensions 3-4-fold in membrane washing medium (adjust volume to $10 \mathrm{~mL}$ ) and ultracentrifuge for $1 \mathrm{~h}$ at $\left(110,000 \times \mathrm{g}, 4^{\circ} \mathrm{C}\right)$. Balance pairs of tubes using membrane washing buffer prior to centrifugation.

3. Carefully aspirate the supernatants using a water pump.

4. Add approximately $100 \mu \mathrm{L}$ of membrane washing buffer (with protease inhibitors) to the envelope pellet. Take an aliquot for determination of protein concentration ${ }^{7}$. Store the purified envelope membrane preparation in liquid nitrogen.

5. Resuspend thylakoids pellet in $3 \mathrm{~mL}$ of membrane washing buffer (with protease inhibitors). Take an aliquot for determination of protein

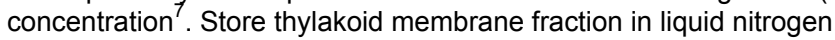

\section{Representative Results}

Successive steps of the procedure resulting in purified chloroplast and their sub-compartments are resumed in Figure 2. The Percoll gradient (Figure 2A) allows distinguishing intact chloroplasts from broken chloroplast and mitochondria (top of the gradient) or nuclei and cell debris (bottom of the gradient). After rupture of the Percoll-purified organelles thanks to an osmotic shock, the resulting fractions are separated on a sucrose gradient (Figure 2B). The stroma (soluble part of the chloroplast) is floating at the surface of the sucrose gradient. The light envelope membrane vesicles are recovered as a discrete yellow band at the $0.6 / 0.93 \mathrm{M}$ sucrose interface. The heaviest thylakoid membranes vesicles are concentrated at the bottom of the tube. After recovery, washing and concentration of the two membrane fractions, proteins are quantified and the composition of all four fractions is analyzed on an SDS-PAGE (Figure 2C). The lanes are loaded on an equal protein basis (20 $\mu \mathrm{g}$ of each purified fraction). Knowing that chloroplasts contain only $1 \%$ of envelope proteins and $50 \%$ of proteins from the stroma or from the thylakoids, this tends to overestimate cross-contamination of purified envelope preparations with other chloroplast sub-compartments. However, this method allows to detect minute amounts of proteins cross-contaminating the envelope fraction. Markers from each compartment (i.e., abundant proteins) are very helpful in evaluating the cross contamination of the fractions. Indeed, the thylakoid and envelope membrane fractions are expected to contain very low amounts of the large subunit of RuBisCO (RBCL), the most abundant protein from the stroma ( $50 \mathrm{kDa})$. Broken chloroplasts can easily be distinguished from intact chloroplast due to the loss of this stromal protein ${ }^{8}$. The light harvesting complex proteins (LHCP) are $25-\mathrm{kDa}$ abundant thylakoid components that should barely (less than $3 \%$ ) contaminate envelope membranes ${ }^{9}$. Finally, the phosphate-triose-phosphate translocator (TPT) is a $30-\mathrm{kDa}$ protein that is only visible in the purified envelope fraction due to its strong enrichment (i.e., 50 to $100 \mathrm{x}$ ) in the envelope fraction when compared to whole chloroplast extracts. Using the method described here, chloroplast sub-compartments are generally poorly cross-contaminated as confirmed using western-blot analyses (Figure 2D) relying on antibodies directed against known markers of all three sub-compartments: the soluble ketol-acid reductoisomerase (KARI) from the stroma, the chloroplast envelope copper ATPase (HMA1), and the light harvesting complex proteins (LHCP) from the thylakoid membranes. Cross-contamination of the three sub-compartments can be quantified using both immunoblotting and mass spectrometry analyses ${ }^{9}$. While the stroma is usually not contaminated by envelope or thylakoid fractions, the purified envelope fractions contain 3\% of thylakoid proteins and up to $10 \%$ of proteins from the stroma. Proteins from the stroma poorly contaminate the thylakoid membranes (less than $1 \%$ ) but thylakoids contain up to $3 \%$ of envelope membrane proteins. More than having a crucial role in identifying the genuine subplastidial location of chloroplast proteins, the present method thus also limits erroneous conclusions about subplastidial localization of proteins resulting from cross contaminations. 


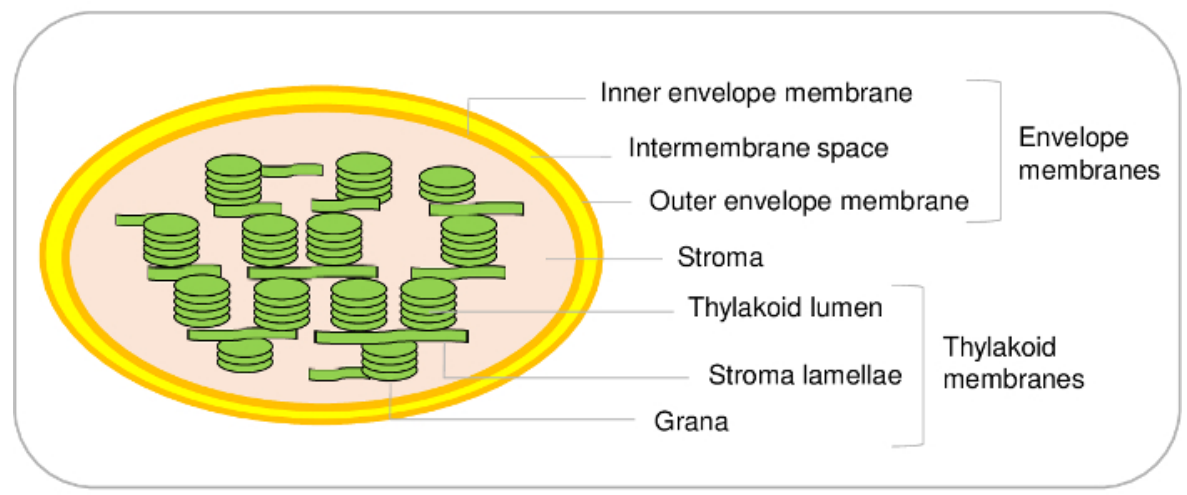

Figure 1: Representative scheme of chloroplast sub-compartments. Please click here to view a larger version of this figure.

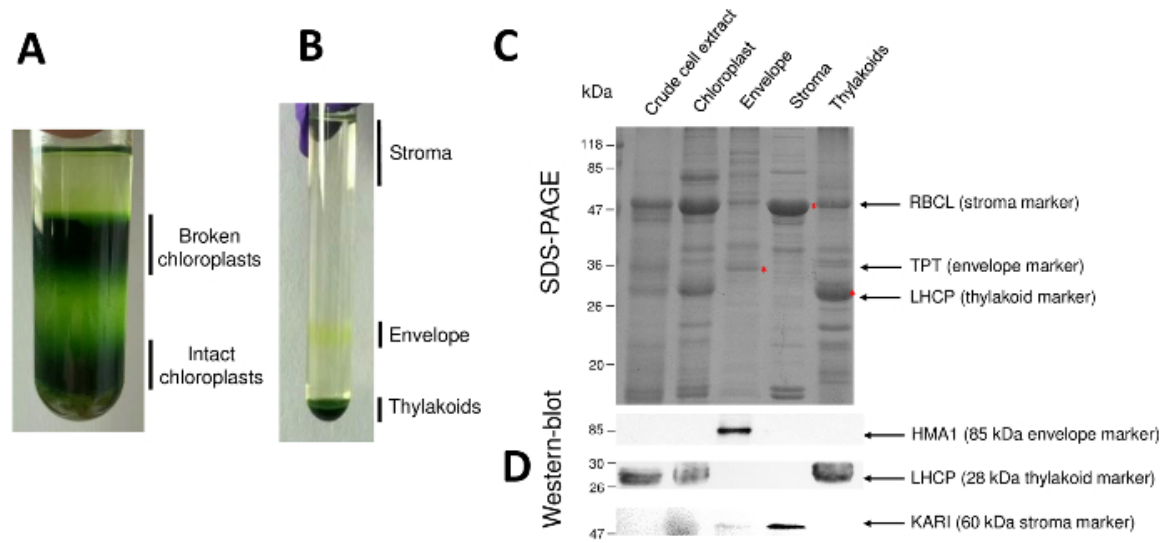

Figure 2: Purification of intact chloroplasts and their three main sub-compartments using Percoll and sucrose gradients. A. Percoll gradient allowing separation of broken and intact chloroplasts. B. Sucrose gradient allowing separation of stroma, envelope, and thylakoid fractions. C. Representative SDS-PAGE of proteins from intact chloroplasts and their three main sub-compartments allowing to visualize abundant markers from each sub-compartment. Each lane contains $10 \mu \mathrm{g}$ of proteins. Molecular weight markers: RBCL, large subunit of RuBisCO (marker for the stroma); TPT, Phosphate/triose-phosphate translocator (marker for the envelope); LHCP, light harvesting complex proteins (marker for the thylakoid). D. Western-blot experiments allowing to detect specific markers (using specific antibodies) from each subcompartment: the chloroplast envelope copper ATPase HMA $1^{10}$, the light harvesting complex proteins LHCP from the thylakoid membranes ${ }^{11}$, and the ketol-acid reductoisomerase KARI from the stroma ${ }^{9}$. Please click here to view a larger version of this figure.

\section{Discussion}

The present article aims to detail the step by step protocol used to purify chloroplasts (and their sub-compartments) from Arabidopsis thaliana. Since the availability of its complete genome sequence almost two decades ago, and of large collections of insertion mutants made available to the community, Arabidopsis is now widely accepted as a model plant. However, while this plant was perfectly adapted for genetic approaches, plant scientists needed to adapt biochemical and physiological tools to this emerging model. Protocols allowing to purify photosynthetically active chloroplasts from leaves of well-established biochemical models like spinach ${ }^{12}$ or pea ${ }^{13}$ thus had to be adapted. The first method describing purification of Arabidopsis chloroplasts was published in $1998^{14}$, just before the release of the Arabidopsis genome sequence. Several years later, simple methods for isolating Arabidopsis chloroplasts compatible with studies aiming to analyze in vitro import of proteins in purified organelles were made available ${ }^{95,16}$. However, these methods did not allow to combine high level of purity and preservation of photosynthetic activity of the purified chloroplasts. More recently ${ }^{17}$, a rapid method was established, which relies on the use of Percoll gradients, and allows to retain almost $90 \%$ of the photosynthesis rate measured in the starting leaves of Arabidopsis.

The protocol described here allows to purify Arabidopsis chloroplasts at an excellent level of purity. Indeed, immunological detection of contaminants from other cell compartments demonstrated that the purified organelles are devoid of mitochondrial and plasma membrane markers $^{9,10}$. This protocol was also efficient to purify chloroplasts from several Arabidopsis ecotypes ${ }^{18}$, like Columbia (Col) or Wassilewskija (WS), i.e., the ecotypes that were used for genome or expressed sequence tags (ESTs) sequencing projects but also to generate T-DNA insertion mutants in Arabidopsis. In other words, when proteomics studies have to be performed, the present protocol is compatible with these two reference ecotypes from Arabidopsis. Finally, the yield of chloroplasts using the present protocol is similar to the one obtained when starting from spinach or pea leaves (i.e., $3 \%$, as measured from the chlorophyll content in the Percoll-purified chloroplast when compared to the total chlorophyll amount present in starting leaves). In terms of proteins, the yield is close to $50 \mathrm{mg}$ of chloroplast proteins, when organelles are purified from $500 \mathrm{~g}$ of 5 -week-old Arabidopsis leaves.

To reach such a good yield (and chloroplast integrity), one should however pay special attention to several steps when using the present protocol. The chloroplast in Arabidopsis is an extremely fragile structure (this is not the case for pea chloroplasts, for example). Specific attention is thus required in order to avoid large-scale rupture of the organelles during purification. The number and size of starch granules present in 
chloroplasts are critical for the preparation of intact chloroplasts. Indeed, chloroplasts containing large starch grain will generally be broken during initial differential centrifugations steps aiming to concentrate the crude chloroplast fractions ${ }^{12}$. Therefore, the plants should be kept overnight in a dark and cold room $\left(4^{\circ} \mathrm{C}\right)$ prior to the experiment, to reduce the amount of starch.

New users of the present protocol could be tempted to start from larger amounts of leaf material (huge rosettes from old Arabidopsis plants with larger leaves) trying to enhance the recovery of purified chloroplasts. However, in our hands, starting from young leaves (5-week-old) is the best compromise to combine yield, purity, and integrity of the purified organelles. Indeed, too old leaves are highly enriched in phenolic compounds that were shown to have a negative impact on chloroplast integrity ${ }^{19}$.

Finally, the initial extraction step (grinding of the tissue) is another critical step. The blending process must be limited to few seconds. As stated above, new users might be tempted to use longer blending, thus expecting to strongly improve the yield of purified organelles. However, if longer blending effectively releases more material from leaves, it appears that the proportion of broken chloroplasts rapidly increases in the crude cell extract. Due to this high ratio of broken to intact chloroplasts in the medium, further purification steps (separation on Percoll gradients) are strongly affected and the yield of the purification is unexpectedly lower.

Availability of specific protocols to purify organelles have allowed a series of high throughput proteomics-based experiments to be conducted on chloroplast samples. These data were made available in several public databases ${ }^{6}$, thus providing to biologists in the field an accurate subcellular (and subplastidial) localization for many chloroplast proteins. This was especially true for envelope proteins whose identity and location remained mostly unknown before these analyses, since envelope membranes represent a minor chloroplast component (1-2\% of the chloroplast proteins) while playing a key role in chloroplast metabolism and biogenesis ${ }^{5,20}$. Using the protocol described here, we recently analyzed the composition of the three main chloroplast compartments from Arabidopsis (i.e., the stroma, the thylakoids, and the envelope membrane system) $)^{9}$. Based on a semi-quantitative proteomics approach (spectral counting), we were able to assess the partitioning of hundreds of proteins in these three chloroplast compartments.

While the present protocol allows to purify the three main compartments of the chloroplast from Arabidopsis, it is also possible to distinguish additional sub-compartments in the chloroplast. Indeed, the envelope membrane system is made of the inner and the outer envelope membranes (Figure 1). However, to the best of our knowledge, a method to purify inner and outer envelope membranes from Arabidopsis

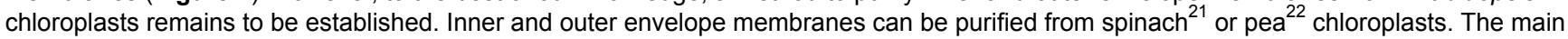
limitation of Arabidopsis mostly results from the limiting amounts of starting material. Starting from $500 \mathrm{~g}$ of Arabidopsis leaves (which already requires $1 \mathrm{~m}^{2}$ surface in a growth chamber) allows purifying only $100 \mu \mathrm{g}$ of envelope proteins. On the other hand, it is easy to start with $5-10 \mathrm{~kg}$ of spinach leaves from the market, to purify large amount of chloroplasts ${ }^{8}$ and to end with a yield of 3 to 10 mg of envelope proteins from this material.

The same is true for thylakoid sub-compartments. Indeed, thylakoids are made of light membrane vesicles (lamellae) and dense structures (grana) (Figure 1). Specific protocols are available to distinguish these two compartments in Arabidopsis ${ }^{23,24}$. Again, based on a quantitative proteomics analysis, we recently inventoried the proteins present in these two sub-compartments ${ }^{24}$. These approaches, together with an indepth investigation of the literature, allowed validating, or proposing hypotheses for, the subplastidial location of hundreds of thylakoid proteins. However, it is important to note that additional membrane microdomains are present at the curved margins of thylakoids. These lipoprotein subcompartments, or plastoglobules, are permanently coupled to thylakoid membranes and contain a specific set of proteins ${ }^{25}$. Using the present protocol, it is thus not possible to distinguish these specific proteins from other thylakoid components.

Some genuine (well-known) envelope, stroma, or thylakoid components are still lacking from the lists of detected proteins. Together with targeted biochemical and immunological analyses, the continual improvement of MS sensitivity will be of great help to revisit the chloroplast content towards a complete repertoire of the composition of its various sub-compartments.

\section{Disclosures}

The authors have nothing to disclose.

\section{Acknowledgements}

This work was supported by a joint PhD fellowship to IB from the INRA Plant Biology and Breeding Division and from the Labex GRAL (ANR-10LABX-49-01). We would also like to acknowledge the ANR project ANR-15-IDEX-02, Dr. Olivier Vallon (IBPC Paris) for antibodies against LHCP and Dr. Renaud Dumas (LPCV, Grenoble) for antibodies against KARI.

\section{References}

1. Zimorski, V., Ku, C., Martin, W. F., Gould, S. B. Endosymbiotic theory for organelle origins. Current Opinion in Microbiology. 22, 38-48 (2014).

2. Gould, S. B., Waller, R. F., McFadden, G. I. Plastid evolution. Annual Review of Plant Biology. 59, $491-517$ (2008).

3. Linka, N., Weber, A. P. Intracellular metabolite transporters in plants. Molecular Plant. 3 (1), 21-53 (2010).

4. Block, M. A., Douce, R., Joyard, J., Rolland, N. Chloroplast envelope membranes: a dynamic interface between plastids and the cytosol. Photosynthesis Research. 92 (2), 225-244 (2007).

5. Rolland, N. et al. The biosynthetic capacities of the plastids and integration between cytoplasmic and chloroplast processes. Annual Review of Genetics. 46, 233-264 (2012).

6. Agrawal, G. K. et al. Plant organelle proteomics: collaborating for optimal cell function. Mass Spectrometry Reviews. 30 (5), $772-853$ (2011).

7. Chua, N.-H. [40] Electrophoretic analysis of chloroplast proteins. Methods in Enzymology. 69, 434-446 (1980).

8. Seigneurin-Berny, D., Salvi, D., Joyard, J., Rolland, N. Purification of intact chloroplasts from Arabidopsis and spinach leaves by isopycnic centrifugation. Current Protocols in Cell Biology. Chapter 3 Unit 330 (2008). 
9. Ferro, M. et al. AT_CHLORO, a comprehensive chloroplast proteome database with subplastidial localization and curated information on envelope proteins. Molecular \& Cell Proteomics. 9 (6), 1063-1084 (2010).

10. Seigneurin-Berny, D. et al. HMA1, a new Cu-ATPase of the chloroplast envelope, is essential for growth under adverse light conditions. Journal of Biological Chemistry. 281 (5), 2882-2892 (2006).

11. Vallon, O. et al. Lateral redistribution of cytochrome b6/f complexes along thylakoid membranes upon state transitions. Proceedings of the National Academy of Sciences of the United States of America. 88 (18), 8262-8266 (1991).

12. Douce, R. J., J. Purification of the chloroplast. M. Edelman, R. Hallick, N. H. Chua (Eds.), Methods in Chloroplast Molecular Biology, Elsevier Science Publishers BV, Amsterdam. 239-256 (1982).

13. Cerovic, Z. G., Plesnicar, M. An improved procedure for the isolation of intact chloroplasts of high photosynthetic capacity. Biochemical Journal. 223 (2), 543-545 (1984).

14. Kunst, L. Preparation of physiologically active chloroplasts from Arabidopsis. Methods in Molecular Biology. 82, 43-48 (1998)

15. Aronsson, H., Jarvis, P. A simple method for isolating import-competent Arabidopsis chloroplasts. FEBS Letters. 529 (2-3), $215-220$ (2002).

16. Ling, Q., Jarvis, P. Analysis of Protein Import into Chloroplasts Isolated from Stressed Plants. Journal of Visualized Experiments. (117) (2016).

17. Seigneurin-Berny, D., Salvi, D., Dorne, A. J., Joyard, J., Rolland, N. Percoll-purified and photosynthetically active chloroplasts from Arabidopsis thaliana leaves. Plant Physiology and Biochemistry. 46 (11), 951-955 (2008).

18. Salvi, D., Rolland, N., Joyard, J., Ferro, M. Purification and proteomic analysis of chloroplasts and their sub-organellar compartments. Methods in Molecular Biology. 432, 19-36 (2008).

19. Walker, D. The use of the oxygen electrode and fluorescence probes in simple measurements of photosynthesis. Oxygraphics Ltd, University of Sheffield, South Yorkshire, UK. (1990).

20. Jarvis, P., Lopez-Juez, E. Biogenesis and homeostasis of chloroplasts and other plastids. Nature Reviews Molecular Cell Biology. 14 (12), 787-802 (2013).

21. Block, M. A., Dorne, A. J., Joyard, J., Douce, R. Preparation and characterization of membrane fractions enriched in outer and inner envelope membranes from spinach chloroplasts. II. Biochemical characterization. Journal of Biological Chemistry. 258 (21), 13281-13286 (1983).

22. Soll, J. Phosphoproteins and protein-kinase activity in isolated envelopes of pea (Pisum sativum L.) chloroplasts. Planta. 166 (3), $394-400$ (1985).

23. Moyet, L., Salvi, D., Tomizioli, M., Seigneurin-Berny, D., Rolland, N. Preparation of Membrane Fractions (Envelope, Thylakoids, Grana, and Stroma Lamellae) from Arabidopsis Chloroplasts for Quantitative Proteomic Investigations and Other Studies. Methods in Molecular Biology. 1696, 117-136 (2018).

24. Tomizioli, M. et al. Deciphering thylakoid sub-compartments using a mass spectrometry-based approach. Molecular \& Cellular Proteomics. 13 (8), 2147-2167 (2014).

25. Spicher, L., Kessler, F. Unexpected roles of plastoglobules (plastid lipid droplets) in vitamin $\mathrm{K} 1$ and E metabolism. Current Opinion in Plant Biology. 25, 123-129 (2015). 